OPEN ACCESS

Edited by: Andrea Gomez-Zavaglia,

Center for Research

and Development in Food

Cryotechnology - Consejo Nacional

De Investigaciones Científicas Y

Técnicas, Argentina

Reviewed by:

Paula Carasi,

Universidad Nacional de La Plata,

Argentina

Anni Woting,

Deutsches Institut für

Ernährungsforschung

Potsdam-Rehbrücke, Germany

${ }^{*}$ Correspondence:

Charles M. A. P. Franz

Charles.Franz@mri.bund.de

Specialty section:

This article was submitted to

Food Microbiology,

a section of the journal

Frontiers in Microbiology

Received: 20 October 2015

Accepted: 20 April 2016

Published: 09 May 2016

Citation:

Cho G-S, Ritzmann F, Eckstein M, Huch M, Briviba K, Behsnilian D,

Neve H and Franz CMAP (2016)

Quantification of Slackia

and Eggerthella spp. in Human Feces

and Adhesion of Representatives

Strains to Caco-2 Cells.

Front. Microbiol. 7:658.

doi: 10.3389/fmicb.2016.00658

\section{Quantification of Slackia and Eggerthella spp. in Human Feces and Adhesion of Representatives Strains to Caco-2 Cells}

\author{
Gyu-Sung Cho' ${ }^{1}$ Felix Ritzmann², Marie Eckstein², Melanie Huch ${ }^{2}$, Karlis Briviba ${ }^{3}$, \\ Diana Behsnilian ${ }^{4}$, Horst Neve ${ }^{1}$ and Charles M. A. P. Franz ${ }^{1 *}$
}

\begin{abstract}
1 Department of Microbiology and Biotechnology, Max Rubner-Institut, Federal Research Institute of Nutrition and Food, Kiel, Germany, ${ }^{2}$ Department of Safety and Quality of Fruit and Vegetables, Max Rubner-Institut, Federal Research Institute of Nutrition and Food, Karlsruhe, Germany, ${ }^{3}$ Department of Physiology and Biochemistry of Nutrition, Max Rubner-Institut, Federal Research Institute of Nutrition and Food, Karlsruhe, Germany, ${ }^{4}$ Department of Food Technology and Bioprocess Engineering, Max Rubner-Institut, Federal Research Institute of Nutrition and Food, Karlsruhe, Germany
\end{abstract}

Eggerthella and Slackia spp. are gut associated bacteria that have been suggested to play roles in host lipid and xenobiotic metabolism. A quantitative PCR method for the selective enumeration of bacteria belonging to either the genus Eggerthella or Slackia was developed in order to establish the numbers of these bacteria occurring in human feces. The primers developed for selective amplification of these genera were tested first in conventional PCR to test for their specificity. Representative species of Eggerthella and Slackia, as well as closely related genera of the Coriobacteriia, were included in the investigation. The selected primers were shown to be capable of specific amplification of species of the genera Eggerthella and Slackia, but not all species of the genera may be amplified by the respective primers. Their use in QPCR experiments to assess the levels of Slackia equolifaciens and Eggerthella lenta in the feces of 19 human volunteers showed they occurred at mean counts of $7 \times 10^{5}$ and $3.1 \times 10^{5} \mathrm{CFU} / \mathrm{g}$ for Eggerthella spp. and Slackia spp., respectively. Electron microscopy investigations showed that while E. lenta cells exhibited slender and very regular shaped rods, Slackia cells showed a remarkably pleomorphic phenotype. Both species did not appear to have fimbriae or pili. Some S. equolifaciens cells showed a characteristic "ribbon" of presumably extracellular material around the cells, particularly at the areas of cell division. The two species also differed markedly in their adhesion behavior to Caco-2 cells in cell culture, as E. lenta DSMZ 15644 showed a high adhesion capacity of $74.2 \%$ adherence of the bacterial cells added to Caco-2 cells, while $S$. equolifaciens DSM $24851^{T}$ on the other hand showed only low adhesion capability, as $6.1 \%$ of bacterial cells remained bound. Speculatively, this may imply that the ecological compartments where these bacteria reside in the gut may be different, i.e., E. lenta may be associated more with the gut wall, while Slackia may be free living in the lumen.

Keywords: coriobacteria, microbiota, gastrointestinal, adherence, cell culture 


\section{INTRODUCTION}

The Coriobacteriia are a diverse group of Gram-positive, nonsporeforming and non-motile bacteria and species belonging to this group of bacteria commonly occur in the human gut. The class Coriobacteriia is one of the deepest branching lineages within the phylum Actinobacteria, branching in the proximity of the phylum Firmicutes (König, 2012; Gupta et al., 2013). Recently, Gupta et al. (2013) proposed a division of the species of the Coriobacteriia into two orders, viz. the Coriobacteriales and Eggerthellales, containing the three families Coriobacteriaceae, Atopobiaceae, and Eggerthellaceae.

Coriobacteria are common members of the human and animal gut microbiota (Eckburg et al., 2005; Arumugam et al., 2011) and amongst these, Collinsella and Eggerthella are among the top five most abundant genera of the phylum Actinobacteria in the human gut. Harmsen et al. (2000) developed 16S rRNA-targeted probes for the coriobacteria and showed that these bacteria are present in human feces in numbers of about $1 \times 10^{10}$ per gram feces. However, the probe used targeted what the authors termed the 'Atopobium cluster' which includes the genera Collinsella, Atopobium, Coriobacterium, and Cryptobacterium. Using this probe, the authors found 'Atopobium-cluster' counts of between $8.6 \times 10^{9}$ up to $1.1 \times 10^{10}$ in human feces (per gram dry weight). Thorasin et al. (2015) further investigated the coverage of the probe and showed that in addition to these, the genera Olsenella, Paraeggerthella, Gordonibacter, and 'Enorma' were also targeted by the probe.

A probe which targeted both the Collinsella and Coriobacterium species ('Collinsella-group' probe) showed lower counts of between $4.1 \times 10^{9}$ to $7.7 \times 10^{9}$ bacteria per gram dry weight feces.

Investigations have shown gut-associated coriobacteria to have a role in gut health. For example, the Coriobacteriaceae have been repeatedly linked to positive effects in host lipid metabolism, and Claus et al. (2011) showed an association between Coriobacteriaceae, particularly bacteria related to Eggerthella lenta and Paraeggerthella hongkongensis on triglyceride levels in mice and concluded that these bacteria are involved in the stimulation of a major hepatic detoxification activity. Strong positive links were also determined with plasma non-highdensity-lipoprotein (non-HDL) in hamsters (Martínez et al., 2009). Martínez et al. (2013) showed that the connection between Coriobacteriaceae and Erysipelothrichaceae and host lipid metabolism could be caused by a metabolic phenotype, i.e., cholesterol excretion, which affects the microbiota. On the other hand, a study which investigated the gut colonization of axenic mice with a typical gut microbiota, and which compared microbiota colonization patterns with hepatic metabolomics profiles, could show that the Coriobacteriaceae are strongly associated with the metabolism of xenobiotics (Claus et al., 2011). Furthermore, Collinsella aerofaciens has been shown to be a member of the core microbiome (Qin et al., 2010).

Eggerthella lenta, Asaccharobacter celatus, Adlercreutzia equolifaciens, and Slackia equolifaciens belong to the Eggerthellaceae and strains of these species have been shown to play a role in the transformation of bioactive secondary plant compounds such as daidzein from soy, or trans-resveratrol from grape vine (Matthies et al., 2009; Tsuji et al., 2012; Bode et al., 2013; Schröder et al., 2013). For example, daidzein was shown to be transformed into dihydrodaidzein and equol by S. isoflavoni-covertans HE8 (Matthies et al., 2009) and the genes involved were cloned and sequenced (Schröder et al., 2013). Equol has a higher binding affinity to human estrogen receptors $\alpha$ and $\beta$ than daidzein (10-80 times) and induces transcription more strongly than any other isoflavone (Decroos et al., 2005). Bode et al. (2013) showed that E. lenta, Adlercreutzia equolifaciens, and $S$. equolifaciens strains produced the metabolite dihydroresveratrol from trans-resveratrol in vitro. The biological activity of this metabolite, and hence the importance of this biotransformation has not yet been well studied. Eggerthella lenta was shown to metabolize the cardiac drug digoxin (Haiser et al., 2013), indicating that members of the gut microbiota may play a role in inactivation of pharmaceuticals in the gut.

Although the numbers of actinobacteria or coriobacteria in the human intestine have been determined before by using oligonucleotide probes (Harmsen et al., 2000), there is currently not much information on specifically the numbers of bacteria belonging to the genera Slackia and Eggerthella spp. in the human intestine, despite their possible importance with respect to production of bioactives in the gut. The aim of this study therefore, was to develop a genus-specific, quantitative PCR method for the specific enumeration of these bacterial genera in human feces. Furthermore, as not much is known about the ecology of these bacteria, the study also aimed to assess whether these bacteria possibly adhere to gut epithelial cells. Lastly, we used scanning and transmission electron microscopy to characterize the cell morphologies.

\section{MATERIALS AND METHODS}

\section{Bacterial Strains and Growth Conditions}

The bacteria and growth media used in this study are shown in Table 1. Apart from coriobacteria belonging to the genera Adlercreutzia, Atopobium, Collinsella, Eggerthella, Enterorhabdus, Gordonibacter, and Slackia, other bacteria used as negative controls for qPCR experiments included Escherichia coli, Lactobacillus plantarum, strains BFE 5092 and 299v, Bacteroides uniformis and Ruminococcus obeum strains (Table 1). All bacterial strains were grown at $37^{\circ} \mathrm{C}$. With the exception of E. coli $\mathrm{DH} 5 \alpha$ and L. plantarum strains, all bacteria were grown anaerobically, either in Hungate tubes under nitrogen (coriobacteria) or in a Don Whitley A45 anaerobic chamber (Shipley, West Yorkshire, UK; Bacteroides uniformis DSM 6597, Ruminococcus obeum DSM 25238) under $80 \% \mathrm{~N}_{2}, 10 \% \mathrm{CO}_{2}$, and $10 \% \mathrm{H}_{2}$.

\section{Scanning and Transmission Electron Microscopy}

Stationary-phase liquid cultures of E. lenta DSMZ 15644 and S. equolifaciens DSMZ $24851^{T}$ grown as described above were used for electron microscopy. Samples were prepared for scanning electron microscopy as described before (Vinderola et al., 
TABLE 1 | Bacteria belonging to the coriobacteria-group, as well as other control strains used in this study.

\begin{tabular}{|c|c|c|}
\hline Strain & Medium & Growth condition \\
\hline Adlercreutzia equolifaciens DSM $19450^{\top}$ & $339^{2}$ & Anaerobic condition ${ }^{1}, 37^{\circ} \mathrm{C}$ \\
\hline Atopobium parvulum DSM $20469^{\top}$ & $104^{3}$ & Anaerobic condition ${ }^{1}, 37^{\circ} \mathrm{C}$ \\
\hline Collinsella intestinalis DSM $13280^{\top}$ & $104^{3}$ & Anaerobic condition ${ }^{1}, 37^{\circ} \mathrm{C}$ \\
\hline Eggerthella lenta DSM 15644 & $339^{2}$ & Anaerobic condition ${ }^{1}, 37^{\circ} \mathrm{C}$ \\
\hline Eggerthella lenta DSM $2243^{\top}$ & $339^{2}$ & Anaerobic condition ${ }^{1}, 37^{\circ} \mathrm{C}$ \\
\hline Enterorhabdus mucosicola DSM $19490^{\top}$ & $339^{2}$ & Anaerobic condition ${ }^{1}, 37^{\circ} \mathrm{C}$ \\
\hline Faecalibacterium prausnitzii DSM 17677 & $339^{2}$ & Anaerobic condition ${ }^{1}, 37^{\circ} \mathrm{C}$ \\
\hline Gordonibacter pamelaeae DSM $19378^{\top}$ & $339^{2}$ & Anaerobic condition ${ }^{1}, 37^{\circ} \mathrm{C}$ \\
\hline Slackia equolifaciens DSM $24851^{\top}$ & $339^{2}$ & Anaerobic condition ${ }^{1}, 37^{\circ} \mathrm{C}$ \\
\hline Escherichia coli DH5alpha & $\mathrm{LB}^{5}$ & Aerobic, $37^{\circ} \mathrm{C}, 180 \mathrm{rpm}^{4}$ \\
\hline Lactobacillus plantarum BFE 5092 & $\mathrm{MRS}^{5}$ & Aerobic, $30^{\circ} \mathrm{C}$ \\
\hline Lactobacillus plantarum 299v & MRS & Aerobic, $30^{\circ} \mathrm{C}$ \\
\hline Bacteroides uniformis DSM $6597^{\top}$ & $104^{3}$ & Anaerobic condition ${ }^{1}, 37^{\circ} \mathrm{C}$ \\
\hline Ruminococcus obeum DSM $25238^{\top}$ & $104^{3}$ & Anaerobic condition ${ }^{1}, 37^{\circ} \mathrm{C}$ \\
\hline
\end{tabular}

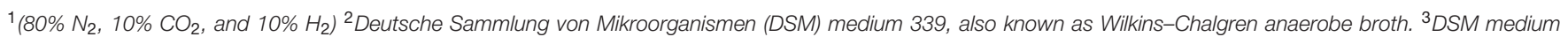

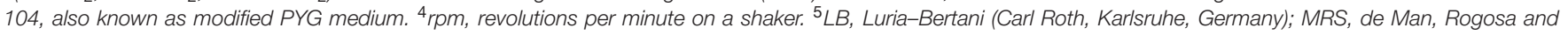
Sharpe (Merck, Darmstadt, Germany).

2012) and viewed in a XL30 scanning electron microscope (FEI Company, Eindhoven) in high vacuum mode with a secondary electron detector at an acceleration voltage of $15 \mathrm{kV}$. Furthermore, S. equolifaciens DSMZ $24851^{T}$ suspensions $\left(10^{7}\right.$ cells $/ \mathrm{mL}$ ) were deposited onto silicon substrates and fixed with $2.5 \%(\mathrm{v} / \mathrm{v})$ glutaraldehyde in $0.1 \mathrm{M}$ sodium phosphate buffer $\mathrm{pH} 7.4$ under anaerobic conditions. The samples were then post-fixed with $0.1 \%(\mathrm{w} / \mathrm{v}) \mathrm{OsO}_{4}$, subjected to an ethanol gradient (25-100\%) and finally critical point dried (BAL-TEC CPD 030, Balzers, Liechtenstein). The silicon substrates with the fixed and dried sample were mounted onto aluminum stubs with double coated carbon conductive tabs. Samples were viewed using a FEG-SEM Quanta 250 (FEI, Czech Republic) equipped with an Everhart-Thornley detector and operated at $10 \mathrm{kV}$.

For transmission electron microscopy, samples were essentially treated as described by Soerensen et al. (2015), with the modification that $2 \%(\mathrm{v} / \mathrm{v})$ glutaraldehyde was used for fixation and $1 \%(\mathrm{w} / \mathrm{v})$ uranyl acetate for negative staining. Electron micrographs were taken using a Tecnai 10 transmission electron microscope (FEI Company, Eindhoven, Netherlands) at an accelerating voltage of $80 \mathrm{kV}$. Digital micrographs were taken with a Megaview G2 CCD camera (Olympus SIS, Münster, Germany).

\section{Genus-Specific Primer Design and Testing by Conventional PCR}

Genus-specific primers were designed for Eggerthella and Slackia genera based on a clustal W alignment of the 16S rRNA gene of the type species shown in Table 1. Clustal W alignment was performed on DNA sequences using the Megalign module [version 10.1.1(3)] of Lasergene (DNASTAR, Inc., Madison, WI, USA). The primers were selected to display maximum sequence difference in 16S rRNA gene nucleotide sequence between the genera in question and the other genera analyzed as controls
(Table 1). The selected primers were then cross-checked in silico as to their specificity by entering them in the ribosomal database project's (RDP) ${ }^{1}$ Seqmatch tool. The settings used to check for the primers were $>1200 \mathrm{bp}$ and $\mathrm{KNN}$ factor 20, quality good). Thus, the primers coriofw ( $5^{\prime}$-GAC GGT ACC TGC AGA AGA AG- $3^{\prime}$ ) and Slackiarev ( $5^{\prime}$-CCC CGG CTT CGA CGG TGC CGC TT- $\left.3^{\prime}\right)$ and Eggfw (5'-TAC TCC TCG CCC CCC TCC TGG-3') and Eggrev (5'-CTT CTT CTG CAG GTA CCG TC$\left.3^{\prime}\right)$ were used to specifically amplify species of the genera Slackia and Eggerthella in conventional PCR experiments, in order to check the specificity of the primers, respectively. The Eggfw primer was located in the V2 region of the 16S rRNA gene from 168 to $188 \mathrm{nt}$ relative to the $16 \mathrm{~S}$ gene of the E. lenta DSM 2243 type strain, while the reverse primer (Eggrev) was located between at 447 and 468 (Supplementary Figure S1). The coriofw primer was located between the V3 and V4 region from 456 to $475 \mathrm{nt}$ relative to the sequence in the Slackia piriformis YIT 12062 genome. The reverse primer (Slackiarev) was found in the V4 region from 570 to 592 nt (Supplementary Figure S1).

DNA for conventional PCR was isolated according the method of Pitcher et al. (1989). The DNA concentration was measured by spectrophotometry and adjusted to $10 \mathrm{ng} / \mu \mathrm{l}$. For each PCR reaction, $100 \mathrm{ng}$ of DNA template, $200 \mathrm{nM}$ of the respective dNTPs, $300 \mathrm{nM}$ of the respective primer, 1x Genaxxon PCR buffer (Genaxxon, Ulm, Germany) containing $6 \mathrm{mM} \mathrm{MgCl}_{2}$ and $1.5 \mathrm{U}$ of Genaxxon Taq polymerase were used. PCR was done in a $50 \mu \mathrm{l}$ volume with an initial degradation step of $94^{\circ} \mathrm{C}$ for $5 \mathrm{~min}$ followed by 32 cycles of $94^{\circ} \mathrm{C}$ for $30 \mathrm{~s}$ denaturing, $62^{\circ} \mathrm{C}$ for $30 \mathrm{~s}$ annealing (for both Slackia and Eggerthella PCR) and $72^{\circ} \mathrm{C}$ for $30 \mathrm{~s}$ elongation. PCR products were subjected to electrophoresis on a $1.8 \%$ agarose gel for $45 \mathrm{~min}$. The gels were stained with Gelred and the specificity of the reaction (presence of a 140 bp band) was confirmed

\footnotetext{
${ }^{1}$ https://rdp.cme.msu.edu/
} 
under UV light using a Fluorchem 5500 (Alpha Innotech, USA).

\section{Quantitative PCR of Slackia and Eggerthella Species}

In order to generate standard curves for quantitative PCR (qPCR) for specific enumeration of Slackia and Eggerthella genera in human feces, DNA was isolated from the representative species S. equolifaciens DSM $24851^{\mathrm{T}}$ and E. lenta strains DSM 15644 and DSM $2243^{\mathrm{T}}$. Before DNA isolation, these bacteria were cultured separately in Wilkins-Chalgren medium (Oxoid, Unipath, Wesel, Germany) containing 0,3 g/L L-cysteine hydrochloride monohydrate (Sigma, Taufkirchen, Germany) and $1 \mathrm{mg} / \mathrm{L}$ resazurin (Sigma) at $37^{\circ} \mathrm{C}$ under anaerobic conditions in Hungate tubes at $37^{\circ} \mathrm{C}$ for $48 \mathrm{~h}$. After $48 \mathrm{~h}$, DNA was isolated from $2 \mathrm{ml}$ of the culture $\left(>1 \times 10^{7} \mathrm{CFU} / \mathrm{ml}\right)$, while $100 \mu \mathrm{l}$ were used to prepare a tenfold dilution series in the anaerobic chamber for determining the viable count by plate counting on WilkinsChalgren agar with L-cysteine hydrochloride monohydrate and resazurin as above. Cell counts were done in triplicate for each culture and were meaned for relating to $\mathrm{qPCR}$ results.

DNA from feces was isolated using the Qiagen mini stool kit as described in a different study (Bode et al., 2013). As our study was not clinical in nature, fecal samples were provided voluntarily by colleagues. The DNA was diluted tenfold and was used as template for qPCR, with the bacterial group specific primers. The standard curves were generated from triplicate cultures of each bacterium, as well as triplicate DNA extractions, each from one of the different cultures. Triplicate Cq-values obtained after qPCR of the standard curve were meaned to obtain a single standard curve. The PCR reaction efficiency $\left(\mathrm{E}=10^{1 /-S}-1\right.$, where $\mathrm{E}=$ efficiency and $\mathrm{S}=$ slope $)$ was calculated from the log-linear part of the standard curve (Töwe et al., 2010).

The qPCR reactions were performed in 96-well plates (BioRad) using a CFX96 quantitative PCR machine (Bio-Rad). The $15 \mu \mathrm{l}$ reaction mix contained $7.5 \mu \mathrm{l}$ of iTaq universal SYBR green supermix (Bio-Rad), $3 \mu \mathrm{l}$ of template DNA, and $200 \mathrm{nM}$ of primers. The annealing temperatures for the qPCR differed for the different genera that were amplified, as this depended on the primers. qPCR amplification conditions were based on a 'two-step' qPCR that included one cycle at $95^{\circ} \mathrm{C}$ for $3 \mathrm{~min}$ for initial denaturation, followed by 40 cycles of denaturing at $95^{\circ} \mathrm{C}$ for $10 \mathrm{~s}$, annealing and extension at the genus-specific temperature at $62^{\circ} \mathrm{C}$ for $1 \mathrm{~min}$. Melting curve analyses were done immediately at the end of the final amplification cycle to determine the specificity of the PCR reaction, by denaturing from 65 to $94^{\circ} \mathrm{C}$ while recording the fluorescence. All qPCRs were repeated three times.

\section{Culturing of Caco-2 Cells in a Transwell System}

Adhesion of S. equolifaciens DSM $24851^{\mathrm{T}}$ and E. lenta strains DSM 15644 to Caco-2 cells were tested in cell culture. For this, Caco-2 cells were seeded at $0.4 \times 10^{6}$ cells onto 9 of 12 transwell filter supports (Costar, Corning, NY, USA), respectively, and grown for 3 weeks with growth medium changes every 2-3 days. Cells were cultured at $37^{\circ} \mathrm{C}$ under $5 \% \mathrm{CO}_{2}$ in Essential Eagle's Medium (Invitrogen, Karlsruhe, Germany), supplemented with $10 \%(\mathrm{v} / \mathrm{v})$ heat-inactivated $\left(56^{\circ} \mathrm{C}, 30 \mathrm{~min}\right)$ fetal calf serum (FCS, PAA Laboratories GmbH, Cölbe, Germany), $100 \mathrm{U} / \mathrm{ml}$ penicillin $\mathrm{G}$ (Invitrogen, Karlsruhe, Germany) and $100 \mu \mathrm{g} / \mathrm{ml}$ streptomycin sulfate (Invitrogen, Karlsruhe, Germany).

Before the adhesion experiments, the Caco-2 cell monolayer was tested for integrity by fluorescein rejection. For this, fluorescein sodium salt (Fluka, Steinheim, Germany) stock solution $(2.5 \mathrm{mg} / 100 \mathrm{ml}$ PBS with Ca and Mg, pH 7.4; Invitrogen, Karlsruhe, Germany) was diluted 1:10 (v/v) with PBS buffer (pH 7.4; Invitrogen). This solution was added to each well of the transwell insert (apical compartment) to measure the transport across the monolayer or the rejection of fluorescein. Three of 12 wells which did not contain cells served as a negative control to measure the free diffusion of fluorescein to the basolateral side of the transwell. Plates were incubated for $40 \mathrm{~min}$ at $37^{\circ} \mathrm{C}$ in the cell culture incubator with $5 \% \mathrm{CO}_{2}$. Then, the concentration of fluorescein was estimated in both the apical and basolateral chambers using a microplate reader (Pherastar BMG Labtech, Ortenberg, Germany); excitation 485, emission $520 \mathrm{~nm}$. All fluorescence measurements were done in triplicate and only inserts with low fluorescein permeability were used for adherence assay, as this indicated that cells were confluent. After determining the integrity of the monolayer, cells were washed twice with $2 \mathrm{ml}$ PBS buffer and then incubated for a further 2 days with cell culture medium without antibiotics.

\section{Adhesion Assays}

All equipment, solutions and plates used for cultivating anaerobic bacteria were pre-incubated in an anaerobic chamber one day prior to the adhesion experiment. Four aliquots of each $2 \mathrm{ml}(\mathrm{v} / \mathrm{v})$ fresh $48 \mathrm{~h}$ culture, of either the Slackia or the Eggerthella strain, were each washed twice by centrifugation at $7500 \times \mathrm{g}$ for $5 \mathrm{~min}$. The pellets were resuspended in $1.5 \mathrm{ml}$ PBS-Ca/Mg. Three of the four $1.5 \mathrm{ml}$ aliquots of bacterial suspension thus prepared were each used to inoculate three wells in a 12 transwell plate used in the adhesion assay, thus serving as a triplicate sample. In addition, the last bacterial suspension was diluted 1:10 in $\mathrm{PBS}-\mathrm{Ca} / \mathrm{Mg}$ in another four aliquots to inoculate an additional three wells (another triplicate sample) and to prepare a further 1:100 dilution in three aliquots which were then used to inoculate a further three wells. Three wells of the 12-well plate were used as control with $\mathrm{PBS}-\mathrm{Ca} / \mathrm{Mg}$ which did not contain bacteria. The plate was incubated for $4 \mathrm{~h}$ under anaerobic conditions $\left(80 \% \mathrm{~N}_{2}\right.$, $10 \% \mathrm{CO}_{2}$, and $10 \% \mathrm{H}_{2}$ ). After $4 \mathrm{~h}$ incubation, the plate was washed with $\mathrm{PBS}-\mathrm{Ca} / \mathrm{Mg}$ twice in both compartments (upper cups and bottom wells) and the Caco-2 cell monolayer in the upper cups, which potentially had bacteria bound to the surface, were detached from the cup membrane with $300 \mu \mathrm{l}$ of a $0.1 \%(\mathrm{w} / \mathrm{v})$ trypsin solution for $5 \mathrm{~min}$ in $37^{\circ} \mathrm{C}$ under anaerobic conditions. These wells were washed two further times by pipetting with $600 \mu \mathrm{l}$ PBS-Ca/Mg followed by centrifugation at $7,500 \times \mathrm{g}$ for $5 \mathrm{~min}$, in order to harvest the Caco- 2 cells 
and bacteria. The pellet was then resuspended in $1 \mathrm{ml}$ PBS $\mathrm{Ca} / \mathrm{Mg}$, serially diluted in $\mathrm{PBS}$ without $\mathrm{Ca} / \mathrm{Mg}$ using a tenfold dilution which was followed by plating onto Wilkins-Chalgren agar with L-cysteine hydrochloride monohydrate and resazurin to enumerate bacteria. These agar plates were incubated in an anaerobic chamber at $37^{\circ} \mathrm{C}$ under anaerobic condition (described above) for $48 \mathrm{~h}$.

\section{RESULTS}

\section{Morphological Characterization by Electron Microscopy}

The morphological characteristics of E. lenta DSMZ 15644 and $S$. equolifaciens DSMZ $24851^{\mathrm{T}}$ are shown in Figure 1. Cells of both cultures were small and were growing in short chains. Both cultures did not reveal flagella. The E. lenta culture was composed of uniformly growing short and thin rods, while $S$. equolifaciens cells exhibited a remarkably pleomorphic phenotype (i.e., irregularly shaped cells of different sizes). As seen at high magnification on transmission and scanning electron micrographs, S. equolifaciens cells revealed characteristically shaped "ribbons" of extracellular material (see arrows in Figure 1, $\mathrm{IIb}, \mathrm{c})$, which are probably synthesized at new cell division regions on the cell wall. No extracellular material was detected on the E. lenta cells.

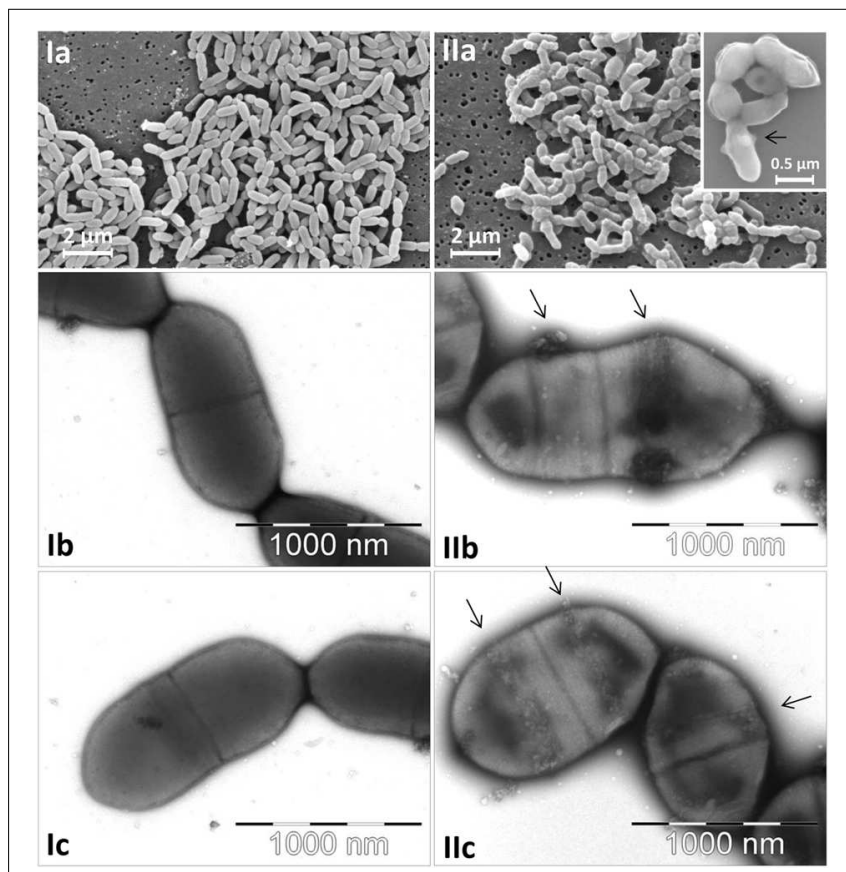

FIGURE 1 | Micrographs of Eggerthella lenta DSMZ 15644 cells (la-c) and of Slackia equolifaciens DSMZ $24851^{\top}$ cells (Ila-c) captured by scanning electron microscopy at low and high magnification (la and Ila with insert) and by transmission electron microscopy at high magnification (lb,c; Ilb,c). The arrows indicate characteristic ribbon bands of extracellular matrix material on the surface of $S$. equolifaciens cells.

\section{Genus-Specific, Conventional PCR for Selective Amplification of Eggerthella spp. and Slackia spp.}

The genus-specific PCR primers for the specific detection of Eggerthella species yielded PCR amplicons of the expected size of $325 \mathrm{bp}$ and is shown for the two Eggerthella reference strains used in this study (Figure 2A). The optimal condition for the Eggerthella genus-specific reaction was at a primer annealing temperature of $60^{\circ} \mathrm{C}$ for $30 \mathrm{~s}$. A PCR product of an expected size of $140 \mathrm{bp}$ was also obtained with the Slackia genus-specific primers when using DNA from S. equolifaciens (Figure 2B). The PCR reaction was also done at a primer annealing temperature of $60^{\circ} \mathrm{C}$. Both genus-specific primers targeted the 16S rRNA gene and were quite specific, as DNA from species of other Actinobacteria genera (e.g., Adlercreutzia, Collinsella, and Gordonibacter) or other gut associated bacteria (Bacteroides, Ruminococcus, Escherichia, Lactobacillus), when used as a matrix, did not yield a PCR product (Figures 2A,B). Only in the case of DNA from Atopobium parvulum DSM 20469 was a, non-specific PCR product of 550 bp, i.e., more than the expected size, detected. The difference in amplicon size between Slackia and Atopobium strains could be easily observed in both normal gel electrophoresis or by a low additional peak in melting curve analysis in qPCR (see below). Thus, the PCR condition and the primers tested for the genus-specific detection and enumeration of Slackia and Eggerthella were deemed acceptable for further qPCR analysis to enumerate these bacteria in feces samples.

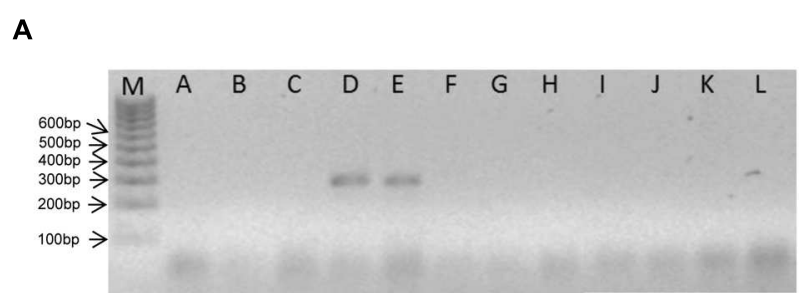

B

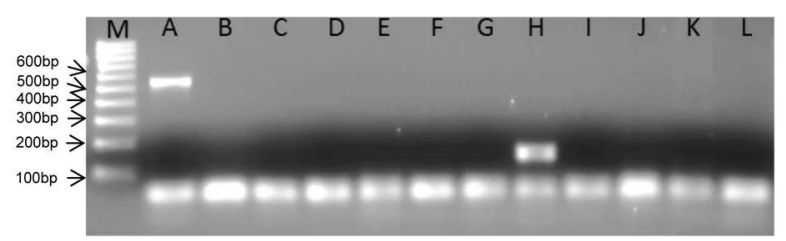

FIGURE 2 | Bands obtained in genus-specific, conventional PCR with Eggerthella (A)- and Slackia (B)- specific primers and DNA from different coriobacterial species. M: 100 bp ladder DNA size marker. A: Atopobium parvulum DSM $20469^{\top}$, B: Adlercreutzia equolifaciens DSM $19450^{\top}$, C: Collinsella intestinalis DSM $13280^{\top}$, D: Eggerthella lenta DSM $2243^{\top}$, E: Eggerthella lenta DSM 15644, F: Enterorhabdus mucosicola DSM $19490^{\top}$, G: Gordonibacter pamelaeae DSM 19378 ${ }^{\top}$, H: Slackia equolifaciens DSM $24851^{\top}$, I: Escherichia coli DH5alpha, J: Faecalibacterium prausnitzii DSM 17677, K: Ruminococcus obeum DSM 25238', L: Lactobacillus plantarum BFE 5092. 

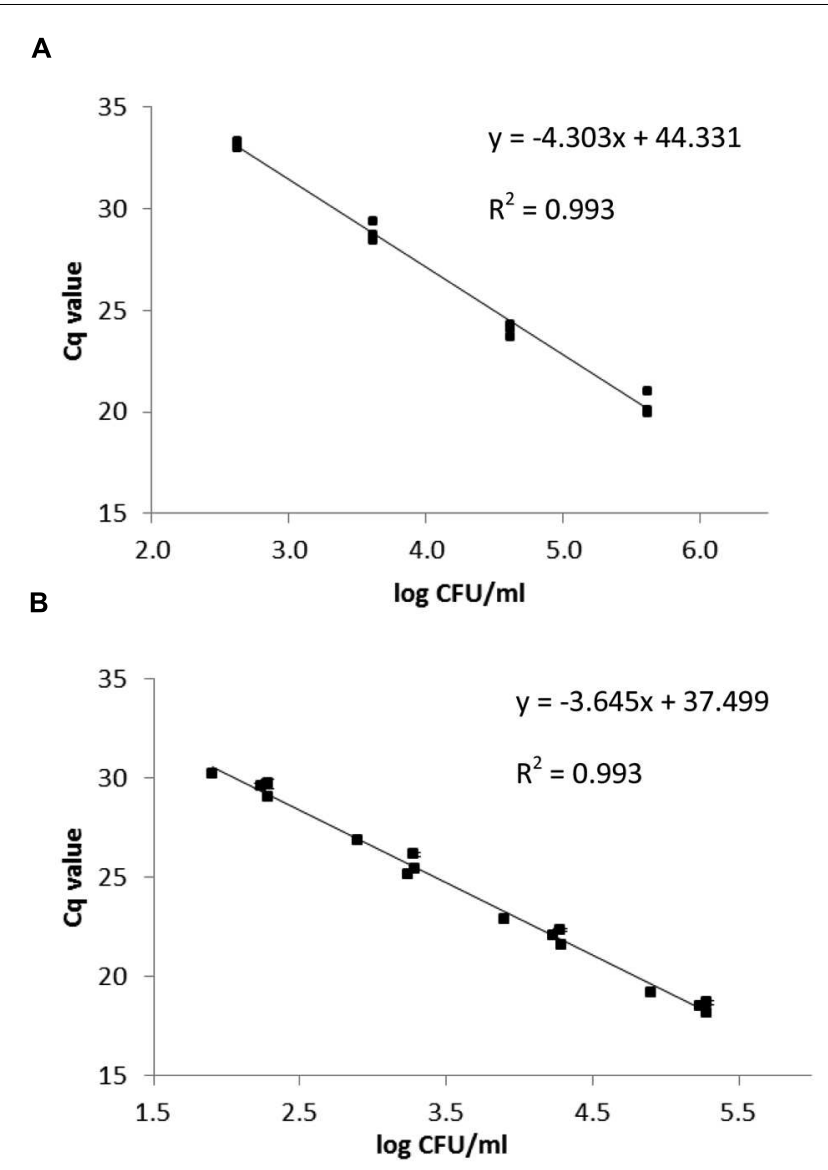

FIGURE 3 | qPCR standard curves relating the Cq-value to log CFU/ml for $E$. lenta strains DSM $2243^{\top}$ and DSM 15644 (A) and S. equolifaciens DSM $24851^{\top}$ (B). The data are based on triplicate independent assessments.

\section{Enumeration of Slackia and Eggerthella Species with qPCR}

Eggerthella lenta DSM $2243^{\mathrm{T}}$, E. lenta DSM 15644 and S. equolifaciens DSM $24851^{\mathrm{T}}$ were grown in pure culture in medium 339 at $37^{\circ} \mathrm{C}$ under anaerobic conditions in order to produce standard curves that relate bacterial counts with Cq-values obtained in the qPCR. The correlations between $\mathrm{Cq}$ values and the $\mathrm{CFU} / \mathrm{ml}$ of Slackia and Eggerthella cell number in standard curves were linear (Figure 3). The standard curve obtained for Eggerthella spp. showed that the minimum number of Eggerthella spp. that could be detected was ca. $4.0 \times 10^{2} \mathrm{CFU} / \mathrm{ml}$ (Figure 3A) and the qPCR efficiency was $70.8 \%$. This PCR efficiency was lower, and may have been a result of the fact that 2 different Eggerthella spp. were used for generating a common standard curve. Alternatively, primer design or salt concentrations may have caused this and could possibly be improved in further studies. The minimum number of Slackia bacteria which could be detected by qPCR was ca. $1 \times 10^{2} \mathrm{CFU} / \mathrm{ml}$ and the qPCR amplification efficiency for the Slackia spp. was $88.1 \%$ (Figure 3B).
In order to determine the number of Slackia and Eggerthella species in feces samples, the human feces DNA from nineteen volunteers obtained in two previous studies (Barth et al., 2012; Bode et al., 2013) was isolated using a DNA mini stool kit (Qiagen) and used as template in qPCR with iTaq SYBRGreen (Bio-Rad). Each bacterial count was done in triplicate and the standard error was determined. The number of bacteria detected with the Eggerthella genus- specific primers ranged from $1.9 \times 10^{3}$ and $7.0 \times 10^{6} \mathrm{CFU} / \mathrm{g}$ feces and the numbers of Slackia bacteria detected with the Slackia-specific primers was observed to range between $1.8 \times 10^{3}$ to $4.0 \times 10^{6} \mathrm{CFU} / \mathrm{g}$ feces, depending on individuals (Table 2). The average Eggerthella and Slackia counts observed for the 19 individuals were $7 \times 10^{5}$ and $3.1 \times 10^{5} \mathrm{CFU} / \mathrm{g}$, respectively, as determined by qPCR (Figure 4). From Table 2 it was noted that 14 of 19 individuals (74\%) and $15 / 19$ (79\%) individuals had counts higher than $1 \times 10^{4} \mathrm{CFU} / \mathrm{g}$ Eggerthella and Slackia spp., respectively. Thus, approximately three-quarters of tested individuals had Eggerthella and Slackia counts of more than $1 \times 10^{4} \mathrm{CFU} / \mathrm{g}$ in their feces, while approx. half of tested individuals had counts of more than $1 \times 10^{5} \mathrm{CFU} / \mathrm{g}$ in the feces.

\section{Adhesion to Caco-2 Cells}

In this study, the adhesion ability of $S$. equolifaciens DSM $24851^{\mathrm{T}}$ and E. lenta DSM 15644, two strict anaerobic bacteria, to intestinal Caco-2 cells in a transwell plate under anaerobic conditions, was determined. E. lenta DSM 15644 showed a high adhesion capacity to Caco-2 cells at a level of $74.2 \%$ adherence (76.9\% in test with bacterial cells diluted $1: 10$ and $71.5 \%$ in test with bacterial cells diluted 1:100; Figure 5). S. equolifaciens DSM $24851^{\mathrm{T}}$ on the other hand showed only low adhesion capacity for adherence to these cells as only $4.7 \%$ (1:10 dilution) and $7.5 \%$ (1:100 dilution) of bacterial cells remained bound and could be recovered after washing of Caco- 2 cells. The probiotic L. plantarum strain $299 \mathrm{v}$ was used as a control, as it is a wellknown probiotic strain previously reported to adhere relatively well (when compared to other probiotic lactic acid bacteria) to Caco-2 cells (Jacobsen et al., 1999) in adhesion assays that were not done in transwell plates. The adhesion capacity of this strain in our study ranged from 2.9 to $4.3 \%$ for the duplicate assessments with either 1:10 or 1:100 diluted bacterial inocula. The differences in percentages between the tests with bacteria from different dilutions are probably the result of deviation in counts between replicate assessments.

\section{DISCUSSION}

Coriobacteria are common members of the human and animal gut microbiota (Eckburg et al., 2005; Arumugam et al., 2011) and amongst these, Collinsella and Eggerthella are among the top five most abundant genera of the phylum Actinobacteria in the human gut. The genus Collinsella by itself even featured among the top six most abundant genera, being present in 32 out of 35 gut microbiota samples investigated at a sequence abundance of $1.8 \%$ of total microbiota (Arumugam et al., 2011). Louis et al. (2007) reported that bacteria of the 
TABLE 2 | Mean number of Eggerthella and Slackia spp. in fecal samples (CFU per gramwet feces) of 19 volunteers.

\begin{tabular}{|c|c|c|c|c|}
\hline Sample from volunteer & Mean no. of Eggerthella spp. (CFU/g feces) & Standard error & Mean of Slackia spp. (CFU/g feces) & Standard error \\
\hline 01 & $2.0 E+03$ & $3.7 E+02$ & $1.0 E+05$ & $1.4 E+03$ \\
\hline 02 & $5.5 E+04$ & $5.2 E+03$ & $7.1 E+05$ & $1.6 E+04$ \\
\hline 03 & $7.0 E+05$ & $6.8 E+04$ & $3.3 E+04$ & $2.7 E+03$ \\
\hline 04 & $1.5 E+04$ & $3.6 E+03$ & $6.7 E+03$ & $2.9 E+03$ \\
\hline 05 & $3.1 E+05$ & $5.2 E+04$ & $1.8 E+03$ & $4.8 E+02$ \\
\hline 06 & $5.6 E+04$ & $2.5 E+03$ & $2.5 E+05$ & $3.3 E+04$ \\
\hline 07 & $1.9 E+03$ & $1.1 E+03$ & $1.3 E+04$ & $1.2 E+03$ \\
\hline 08 & $9.7 E+03$ & $8.9 E+02$ & $1.2 E+05$ & $1.5 E+04$ \\
\hline 09 & $3.8 E+03$ & $5.5 E+02$ & $2.3 E+03$ & $1.5 E+03$ \\
\hline 10 & $2.5 E+04$ & $1.8 E+03$ & $4.0 E+06$ & $4.9 E+05$ \\
\hline 11 & $7.0 E+06$ & $2.1 E+05$ & $1.4 E+05$ & $9.8 E+03$ \\
\hline 12 & $4.2 E+05$ & $6.2 E+03$ & $3.4 E+04$ & $3.3 E+03$ \\
\hline 13 & $7.1 E+05$ & $4.8 E+03$ & $2.1 E+05$ & $8.4 E+03$ \\
\hline 14 & $3.9 E+06$ & $2.2 E+05$ & $4.0 E+04$ & $3.2 E+03$ \\
\hline 15 & $6.6 E+03$ & $3.9 E+03$ & $4.6 E+04$ & $2.5 E+03$ \\
\hline 16 & $1.6 E+04$ & $2.3 E+03$ & $2.4 E+04$ & $4.9 E+03$ \\
\hline 17 & $8.2 E+04$ & $5.1 E+03$ & $1.1 E+05$ & $9.8 E+04$ \\
\hline 18 & $5.0 E+05$ & $3.9 E+04$ & $2.0 E+04$ & $1.2 E+03$ \\
\hline 19 & $2.1 E+04$ & $3.4 E+03$ & $8.9 E+03$ & $1.2 E+03$ \\
\hline
\end{tabular}

The standard error of triplicate counts is indicated.

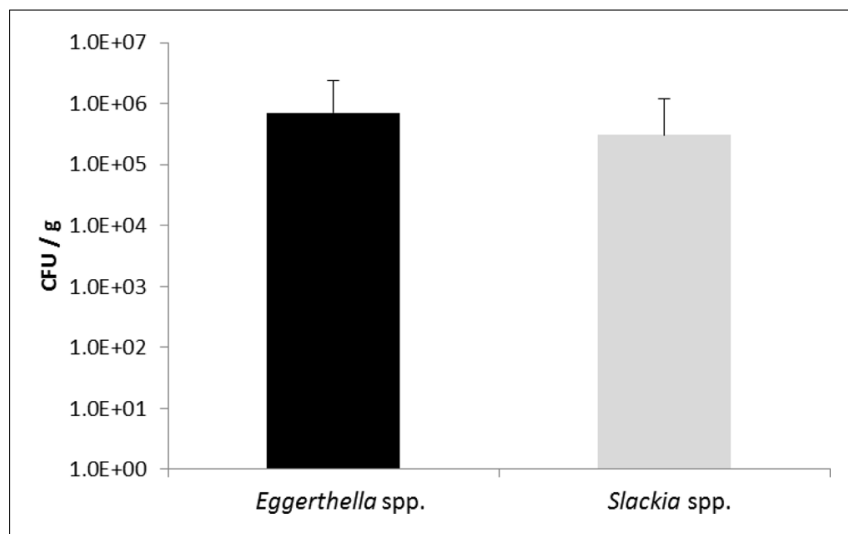

FIGURE 4 | Bacterial counts calculated from triplicate qPCR of all 19 individual human feces samples. The standard deviation is shown. Black bar: mean values of Eggerthella spp., gray bar: mean values of Slackia spp.

Atopobium cluster, belonging to the Atopobiaceae of the order Coriobacteriales, occur at an abundance of 2.1 to $11.9 \%$ of total bacteria of colonic bacteria. The Actinobacteria, however, have in numerous studies been described to be underrepresented or to remain undetected in clone libraries of the human gut microbiota (Wilson and Blitchington, 1996; Suau et al., 1999; Harmsen et al., 2000; Koenig et al., 2011; Maukonen et al., 2012; Sim et al., 2012; Thorasin et al., 2015). Possible explanations for this may be the relative hydrophobicity of the cell walls and high mol\% G+C content in the DNA, which leads to difficulties with DNA isolation and PCR amplification of clone libraries due to bias in PCR primers (Thorasin et al., 2015).

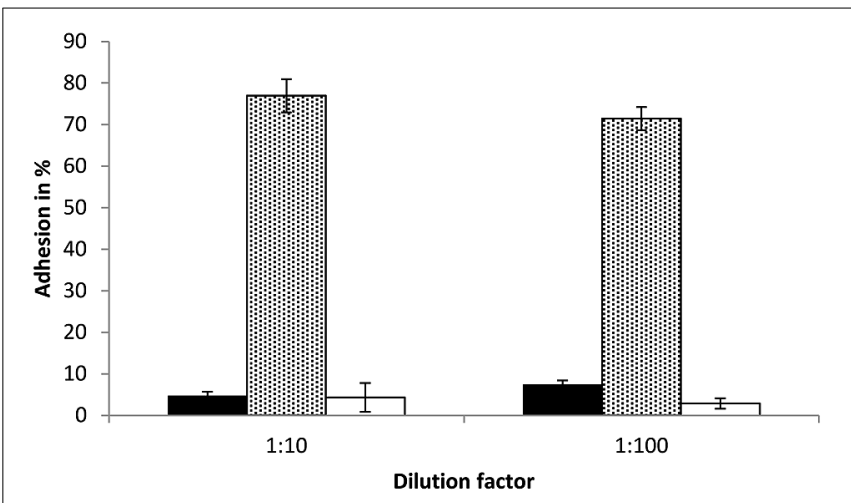

FIGURE 5 | Bacterial adhesion (\%) to Caco-2 cells in transwell plates as determined in triplicate independent analyses. Black bar:

S. equolifaciens DSM $24851^{\top}$, dotted gray bar: E. lenta DSM 15644, white bar: Lactobacillus plantarum 299v (control probiotic strain). The error bar indicates the standard deviation.

This study aimed to develop a quantitative PCR assay specific for the genera Slackia and Eggerthella. For this, primers were developed based on an in silico approach and tested for their relative specificities. The primers and amplification conditions developed proved quite specific for specific amplification of the genera Slackia and Eggerthella, for which so far, no specific PCR amplification primers have been developed. When the same primers were used for quantitative PCR, a standard curve was first established with pure cultures of $S$. equolifaciens and E. lenta for which PCR signals were correlated with viable cell counts. A previous qPCR study based on enumeration of bacteria from pure cultures showed that the qPCR efficiencies of the reactions ranged from 80 to $87 \%$ in different dilutions of the matrix 
DNA (Töwe et al., 2010). These amplification efficiencies were considered suitable for deriving bacterial cell counts from qPCR standard curve data (Töwe et al., 2010); therefore, the similar amplification efficiencies determined in this study, also indicated the suitability of the standard curves based on the 16S rRNA gene primers for enumeration of Slackia and Eggerthella species by qPCR for determining genus-specific bacterial counts in this study.

Previous studies on numbers of coriobacteria in human feces which were based on FISH using a 'Atopobium cluster'-specific probe, which targets the genera Collinsella, Coriobacterium, Atopobium, and Cryptobacterium, showed that these bacteria occur in the feces of humans at levels above ca. $5 \times 10^{9}$ up to $1.1 \times 10^{10}$ per gram dry weight (Harmsen et al., 2000; Thorasin et al., 2015). However, these studies did not provide any information on the specific numbers of Slackia and Eggerthella bacteria present in the feces, as the probes used in the FISH experiments did not hybridize to bacteria of these genera. As these bacteria, however, may play an important role in the metabolism of bioactive compounds such as daidzein and resveratrol (Matthies et al., 2009; Bode et al., 2013), this study aimed to determine their numbers in human feces. Our results show that the numbers of Slackia and Eggerthella were considerably lower than the reported numbers of the 'Atopobium'- or Collinsella-groups (Harmsen et al., 2000; Thorasin et al., 2015), at an average of about 105 to106 CFU/g feces. Tsuji et al. (2010) developed a Slackia primer based on the 16S rRNA sequence of the Slackia sp. strain NATTS, which not only covered strain NATTS, but also related strains. When this primer was used, they were able to enumerate this Slackia sp. at a population level of log 6.4 cells per gram wet feces (Tsuji et al., 2010). This is similar to the numbers found in this study. For some individuals, the numbers of these bacteria determined in our study were much lower at levels of about $10^{3}$ CFU/g. However, in the case of our Slackia-specific primers, the RDP Sequence match showed that these were specific only for S. equolifaciens, S. piriformis and S. faecicanis and thus did not cover the complete Slackia genus. Thus these primers could have missed S. isoflavoni-convertans, which was enumerated in the study by Tsuji et al. (2010). The use of the primers in this study may therefore possibly underreport the numbers of all Slackia spp. in human feces. It should be considered, however, that the extent of underreporting would be difficult to judge, especially as the Slackia reverse primer, which is responsible for the specificity of the amplification, differed in only two nucleotides out of the $23 \mathrm{nt}$ primer. This primer in conventional PCR did yield a specific product. On the other hand, the primers also amplified Atopobium parvulum, and where thus not strictly genus specific either. Clearly, this should be more closely investigated with more strains, also related, non-Slackia strains, in a further study.

Schwiertz et al. (2000) used a rRNA oligonucleotide probe to determine the numbers of E. lenta in human feces and found these bacteria to be present at a range of $\log 8.1-8.5$. This is clearly higher than the counts determined in this study, which ranged from $\log 3$ to $\log 6$. Reason for this may be that the probe used by Schwiertz et al. (2000) detected also other Eggerthella species, which at the time had not been described or investigated, as in the report of Schwiertz et al. (2000) the Eggerthella lenta was taxonomically still considered as Eubacterium lentum. The Eggfw/Eggrev primers used in this study according to the RDP Sequence match targeted Eggerthella lenta and E. sinensis. Thus the primer pair used in our study may also underreport the amounts of Eggerthella in human feces samples. As for the case of the Slackia primers, the specificity should also be investigated with more Eggerthella species and other related, non-Eggerthella species, in more detailed further studies. Furthermore, the Eggerthella PCR products in the conventional PCR showed weaker bands when compared to the band obtained with the Slackia primers, therefore the PCR conditions could still be optimized further for the Eggerthella PCR.

Thorasin et al. (2015) showed that the human fecal 'Atopobium-cluster' population, especially species of the genera Collinsella, Gordonibacter, Olsenella, and Paraeggerthella, was quite stable over a three months period. However, these data did not reveal whether species of the genera Slackia and/or Eggerthella would show a similar stable composition. As these occur in lower numbers, this might hypothetically not be the case. Furthermore, the numbers of Slackia and Eggerthella in our study seem to vary considerable from between $10^{3}$ to $10^{6}$ $\mathrm{CFU} / \mathrm{g}$ in different individuals in this study, indicating that the health-associated functions of these bacteria, e.g., in converting daidzein to equol, may be functioning at different levels of effectiveness in different individuals that contain these bacteria in their microbiota. Given the relative importance of these bacteria in gastrointestinal health, this clearly would warrant further investigation.

The adhesion assays with Caco-2 cells in this study showed that cells in the transwell plates grew well to reach confluence within 21 days and that $1: 10$ or 1:100 dilutions of the bacterial cultures were suitable for use in binding assays. The novelty of this study was that incubation of Caco-2 cells with bacteria was done under strictly anaerobic conditions and using differentiated polarized Caco-2 cells on permeable membranes. These conditions are similar to an in vivo situation: Bacteria were applied to the apical side of Caco- 2 cells, but the basolateral side of the cells was incubated with the bacteria-free basolateral compartment medium. Furthermore, the integrity and differentiation of Caco- 2 cells were determined by fluorescein (a compound known to have low permeability across the integral Caco-2 cell monolayer) and measurement of transepithelial electrical resistance (Masungi et al., 2004), as well as electronic microscopy (formation of microvilli; SEM as done in this study with similar method). Our pilot experiments done with Caco-2 cells incubated under anaerobic conditions without bacteria for $4 \mathrm{~h}$ resulted in only a slight decrease in viability of Caco-2 cells as tested by trypan blue exclusion (results not shown). This is the reason for incubating the bacteria on cells during the adhesion studies for $4 \mathrm{~h}$. The results showed that L. plantarum 299v showed low binding to Caco-2 cells in comparison with Eggerthella bacteria (Figure 4). Although previously described to bind well, the adhesion behavior in this study may be different because of differences in methodology to determine binding between the two studies. Jacobsen et al. (1999) counted bacteria in 20 microscopic fields, instead of determining 
the percentage of bacteria adhering by viable plate counting, as was done in this study. In addition, the ratio of bacteria/Caco-2 cells used may have been different to that used in this study.

Eggerthella lenta showed high adhesion of up to $80 \%$ to gut epithelial cells in cell culture in this study, while the binding ability of $S$. equolifaciens was low, as only less than $10 \%$ of cells bound to Caco- 2 cells. We recently sequenced the genome of S. equolifaciens DSM $24851^{\mathrm{T}}$ at a commercial sequencing facility that used an Illumina Miseq for sequencing. The sequence data were aligned into contigs and scaffolds and sent for annotation using the RAST (Rapid Annotation using Subsystem Technology) server (Aziz et al., 2008). There were still numerous gaps in the chromosomal sequence, and more bioinformatic analyses need to be performed, but preliminary results show that $S$. equolifaciens DSM $24851^{\mathrm{T}}$ does not possess genes for fimbriae or pili, which was also evident from our electron microscopy results. Interestingly, on the cells extracellular material arranged in "ribbons" could be seen, especially in areas where the cell was dividing, suggesting the presence of extracellular matrix proteins coating the cells (Figure 1, IIa insert). Indeed, in the genome sequence of S. equolifaciens, genes for extracellular matrix components were present.

This study is the first to report on the approximate numbers of coriobacteria belonging to the genera Slackia and Eggerthella, especially the species S. equolifaciens and E. lenta for which the

\section{REFERENCES}

Arumugam, M., Raes, J., Pelletier, E., Le Paslier, D., Yamada, T., Mende, D. R., et al. (2011). Enterotypes of the human gut microbiome. Nature 473, 174-180. doi: 10.1038 /nature09944

Aziz, R. K., Bartels, D., Best, A. A., DeJongh, M., Disz, T., Edwards, R. A., et al. (2008). The RAST server: rapid annotation using subsystem technology. BMC Genomics 9:75. doi: 10.1186/1471-2164-9-75

Barth, S. W., Koch, T. C., Watzl, B., Dietrich, H., Will, F., and Bub, A. (2012). Moderate effects of apple juice consumption on obesity-related markers in obese men: impact of diet-gene interaction on body fat content. Eur. J. Nutr. 51, 841-850. doi: 10.1007/s00394-011-0264-6

Bode, L., Bunzel, D., Huch, M., Cho, G. S., Ruhland, D., Bunzel, M., et al. (2013). In vivo and in vitro metabolism of trans-resveratrol by human gut microbiota. Am. J. Clin. Nutr. 97, 295-309. doi: 10.3945/ajcn.112.049379

Claus, S. P., Ellero, S. L., Berger, B., Krause, L., Bruttin, A., Molina, J., et al. (2011). Colonization-induced host-gut microbial metabolic interaction. MBio 2, e271-10. doi: 10.1128/mBio.00271-10

Decroos, K., Vanhemmens, S., Cattoir, S., Boon, N., and Verstraete, W. (2005). Isolation and characterization of an equol-producing mixed microbial culture from a human faecal sample and its activity under gastrointestinal conditions. Arch. Microbiol. 183, 45-55. doi: 10.1007/s00203-0040747-4

Eckburg, P. B., Bik, E. M., Bernstein, C. N., Purdom, E., Dethlefsen, L., Sargent, M., et al. (2005). Diversity of the human intestinal microbial flora. Science 308, 1635-1638. doi: 10.1126/science.1110591

Gupta, R. S., Chen, W. J., Adeolu, M., and Chai, Y. (2013). Molecular signatures for the class Coriobacteriia and its different clades; proposal for division of the class Coriobacteriia into the emended order Coriobacteriales, containing the emended family Coriobacteriaceae and Atopobiaceae fam. nov., and Eggerthellales ord. nov., containing the family Eggerthellaceae fam. nov. Int. J. Syst. Evol. Microbiol. 63, 3379-3397. doi: 10.1099/ijs.0.048 371-0

Haiser, H. J., Gootenberg, D. B., Chatman, K., Sirasani, G., Balskus, E. P., and Turnbaugh, P. J. (2013). Predicting and manipulating cardiac drug inactivation primers were more specific. These are bacteria which may play an important role in the gut health of human beings. Our results also suggest a distribution of these species to specific compartments, i.e., the gut lumen in the case of Slackia and the gut wall in the case of Eggerthella. However, basing this assumption on adhesion data in cell culture would be speculative, and thus this aspect should be further investigated.

\section{AUTHOR CONTRIBUTIONS}

G-SC planned and carried out experiments on qPCR, cell culture and adhesion of coriobacteria. DB and HN carried out TEM and REM, respectively. FR carried out adhesion experiments in cell culture with G-SC. ME carried out qPCR with G-SC. MH cultivated bacteria anaerobically. KB developed the anaerobic cell culture with G-SC and tested cell survival. CF designed and was responsible for research with G-SC and also designed custom primers for qPCR with G-SC and ME.

\section{SUPPLEMENTARY MATERIAL}

The Supplementary Material for this article can be found online at: http://journal.frontiersin.org/article/10.3389/fmicb. 2016.00658

by the human gut bacterium Eggerthella lenta. Science 341, 295-298. doi: $10.1126 /$ science. 1235872

Harmsen, H. J. M., Wildeboer-Veloo, A. C. M., Grijpstra, J., Knol, J., Degener, J. E., and Welling, G. W. (2000). Development of 16S rRNA-based probes for the Coriobacterium group and the Atopobium Cluster and their application for enumeration of Coriobacteriaceae in human feces from volunteers of different age groups. Appl. Environ. Microbiol. 66, 4523-4527. doi: 10.1128/AEM.66.10.4523-4527.2000

Jacobsen, C. N., Rosenfeldt Nielsen, V., Hayford, A. E., Moller, P. L., Michaelsen, K. F., Paerregaard, A., et al. (1999). Screening of probiotic activities of fortyseven strains of Lactobacillus spp. by in vitro techniques and evaluation of the colonization ability of five selected strains in humans. Appl. Environ. Microbiol. 65, 4949-4956.

Koenig, J. E., Spor, A., Scalfone, N., Fricker, A. D., Stombaugh, J., Knight, R., et al. (2011). Succession of microbial consortia in the developing infant gut microbiome. Proc. Natl. Acad. Sci. U.S.A. 108(Suppl. 1), 4578-4585. doi: $10.1073 /$ pnas. 1000081107

König, H. (2012). "Class III. Coriobacteriia class. nov," in Bergey's Manual of Systematic Bacteriology, eds M. Goodfellow, P. Kämpfer, H. J. Busse, M. E. Trujillo, K. Suzuki, W. Ludwig, et al. (New York, NY: Springer), 1975-1976.

Louis, P., Scott, K. P., Duncan, S. H., and Flint, H. (2007). Understanding the effects of diet on bacterial metabolism in the large intestine. J. Appl. Microbiol. 102, 1197-1208. doi: 10.1111/j.1365-2672.2007.03322.x

Martínez, I., Muller, C. E., and Walter, J. (2013). Long-term temporal analysis of the human fecal microbiota revealed a stable core of dominant species. PLOS ONE 8:e69621. doi: 10.1371/journal.pone.0069621

Martínez, I., Wallace, G., Zhang, C., Legge, R., Benson, A. K., Carr, T. P., et al. (2009). Diet-induced metabolic improvements in a hamster model of the hypercholesterolemia are strongly linked to alterations of the gut microbiota. Appl. Environ. Microbiol. 75, 4175-4184. doi: 10.1128/AEM. 00380-09

Masungi, C., Borremans, C., Willems, B., Mensch, J., van Dijck, A., Augustijns, P., et al. (2004). Usefulness of a novel Caco-2 cell perfusion system. I. In vitro prediction of the absorption potential of passively diffused compounds. J. Pharm. Sci. 93, 2507-2521. doi: 10.1002/jps.20149 
Matthies, A., Blaut, M., and Braune, A. (2009). Isolation of a human intestinal bacterium capable of daidzein and genistein conversion. Appl. Environ. Microbiol. 75, 1740-1744. doi: 10.1128/AEM.01795-08

Maukonen, J., Simoes, C., and Saarela, M. (2012). The currently used commercial DNA-extraction methods give different results of clostridial and actinobacterial populations derived from human fecal samples. FEMS Microbiol. Ecol. 79, 697-708. doi: 10.1111/j.1574-6941.2011.01257.x

Pitcher, D. G., Saunders, N. A., and Owen, R. J. (1989). Rapid extraction of bacterial genomic DNA with guanidinium thiocyanate. Lett. Appl. Microbiol. 8, 151-156. doi: 10.1111/j.1472-765X.1989.tb00262.x

Qin, J., Li, R., Raes, J., Arumugam, M., Burgdorf, K. S., Manichanh, C., et al. (2010). A human gut microbial gene catalogue established by metagenomic sequencing. Nature 464, 59-65. doi: 10.1038/nature08821

Schröder, C., Matthies, A., Engst, W., Blaut, M., and Braune, A. (2013). Identification and expression of genes involved in the conversion of daidzein and genistein by the equol-forming bacterium Slackia isoflavoniconvertens. Appl. Environ. Microbiol. 79, 3494-3502. doi: 10.1128/AEM.03693-12

Schwiertz, A., Le Blay, G., and Blaut, M. (2000). Quantification of different Eubacterium spp. in human fecal samples with species-specific 16S rRNAtargeted oligonucleotide probes. Appl. Environ. Microbiol. 66, 375-382. doi: 10.1128/AEM.66.1.375-382.2000

Sim, K., Cox, M. J., Wopereis, H., Martin, R., Knol, J., Li, M. S., et al. (2012). Improved detection of bifidobacteria with optimised 16S rRNAgene based pyrosequencing. PLoS ONE 7:e32543. doi: 10.1371/journal.pone. 0032543

Soerensen, M. C. H., Gencay, Y. E., Birk, T., Baldvinsson, S. B., Jaeckel, C., Hammerl, J. A., et al. (2015). Primary isolation strain determines both phage type and receptors recognised by Campylobacter jejuni bacteriophages. PLoS ONE 10:e0116287. doi: 10.1371/journal.pone.0116287

Suau, A., Bonnet, R., Sutren, M., Godon, J. J., Gibson, G. R., Collins, M. D., et al. (1999). Direct analysis of genes encoding 16S rRNA from complex communities reveals many novel molecular species within the human gut. Appl. Environ. Microbiol. 65, 4799-4807.
Thorasin, T., Hoyles, L., and McCartney, A. L. (2015). Dynamics and diversity of the 'Atopobium cluster'in human faecal microbiota, and phenotypic characterization of 'Atopobium cluster' isolates. Microbiology 161, 565-579. doi: 10.1099/mic.0.000016

Töwe, S., Kleineidam, K., and Schloter, M. (2010). Differences in amplification efficiency of standard curves in quantitative realtime PCR assays and consequences for gene quantification in environmental samples. J. Microbiol. Methods 82, 338-341. doi: 10.1016/j.mimet.2010.07.005

Tsuji, H., Moriyama, K., Nomoto, K., and Akaza, H. (2012). Identification of an enzyme system for daidzein-to-equol conversion in Slackia sp. strain NATTS. Appl. Environ. Microbiol. 78, 1228-1236. doi: 10.1128/AEM. 06779-11

Tsuji, H., Moriyama, K., Nomoto, K., Miyanaga, N., and Akaza, H. (2010). Isolation and characterization of the equol producing bacterium Slackia sp. strain NATTS. Arch. Microbiol. 192, 279-287. doi: 10.1007/s00203-010-0546-Z

Vinderola, G., Zacarias, M. F., Bockelmann, W., Neve, H., Reinheimer, J., and Heller, K. J. (2012). Preservation of functionality of Bifidobacterium animalis subsp. lactis INL1 after incorporation of freeze-dried cells into different food matrices. Food Microbiol. 30, 274-280. doi: 10.1016/j.fm.2011.12.004

Wilson, K. H., and Blitchington, R. B. (1996). Human colonic biota studied by ribosomal DNA sequence analysis. Appl. Environ. Microbiol. 62, 2273-2278.

Conflict of Interest Statement: The authors declare that the research was conducted in the absence of any commercial or financial relationships that could be construed as a potential conflict of interest.

Copyright (c) 2016 Cho, Ritzmann, Eckstein, Huch, Briviba, Behsnilian, Neve and Franz. This is an open-access article distributed under the terms of the Creative Commons Attribution License (CC BY). The use, distribution or reproduction in other forums is permitted, provided the original author(s) or licensor are credited and that the original publication in this journal is cited, in accordance with accepted academic practice. No use, distribution or reproduction is permitted which does not comply with these terms. 